\title{
Predictive systems models can help elucidate bee declines driven by multiple combined stressors
}

\author{
Mickaël Henry $^{1,2}$, Matthias A. Becher ${ }^{3}$, Juliet L. Osborne ${ }^{3}$, Peter J. Kennedy ${ }^{3}$, \\ Pierrick Aupinel ${ }^{4}$, Vincent Bretagnolle ${ }^{5,6}$, François Brun ${ }^{7}$, Volker GrimM ${ }^{8}$, \\ Juliane $\operatorname{HORN}^{8}$, Fabrice REQUIER ${ }^{1,2}$ \\ ${ }^{1}$ INRA, UR406 Abeilles et Environnement, F-84914, Avignon, France \\ ${ }^{2}$ UMT Protection des Abeilles dans l'Environnement, Site Agroparc, F-84914, Avignon, France \\ ${ }^{3}$ Environment and Sustainability Institute, University of Exeter, Penryn Campus, Penryn, Cornwall TR10 9FE, UK \\ ${ }^{4}$ INRA, UE1255, UE Entomologie, F-17700, Surgères, France \\ ${ }^{5}$ Centre d'Etudes Biologiques de Chizé, UMR 7372, CNRS \& Université de La Rochelle, F-79360, Beauvoir-sur-Niort, \\ France \\ ${ }^{6}$ LTER Zone Atelier Plaine \& Val de Sèvre, Centre d'Etudes Biologiques de Chizé, CNRS, F-79360, Villiers-en-Bois, \\ France \\ ${ }^{7}$ ACTA INRA, UMR 1248 AGIR, F-31326, Castanet Tolosan cedex, France \\ ${ }^{8}$ UFZ, Helmholtz Centre for Environmental Research - UFZ, Permoserstr. 15, DE-04318, Leipzig, Germany
}

Received 30 March 2016 - Revised 4 August 2016 - Accepted 22 September 2016

\begin{abstract}
Bee declines are driven by multiple combined stresses, making it exceedingly difficult to identify experimentally the most critical threats to bees and their pollination services. We highlight here the too often ignored potential of mechanistic models in identifying critical stress combinations. Advanced bee models are now available as open access tools and offer an unprecedented opportunity for bee biologists to explore bee resilience tipping points in a variety of environmental contexts. We provide general guidelines on how to run bee models to help detect a priori critical stress combinations to be targeted in the field. This so-called funnel analysis should be performed in tight conjunction with the recent development of large-scale field monitoring programs for bee health surveillance.
\end{abstract}

\section{Apis mellifera / field monitoring program / honeybees / mechanistic modeling / agent-based models}

\section{INTRODUCTION}

The early reviews on the causes of pollinator declines (Potts et al. 2010; Vanbergen and the Insect Pollinators Initiative 2013) highlighted the potential role of multiple stressor interactions and synergies. Recently, Goulson et al. (2015) updated the state of knowledge on combined stressors on bees, reviewing evidence from in vitro and in vivo experiments. They concluded that disentangling the effects of multiple combined stressors is

Corresponding author: M. Henry, mickael.henry@paca.inra.fr

Manuscript editor: David Tarpy exceedingly difficult, implying that evidence will not appear rapidly enough given the urgency of the situation. Here, we would like to draw bee biologists' attention to an under-used shortcut that may help bypass some technical barriers, namely the in silico pathway (Evans et al. 2013; Stillman et al. 2015). Mechanistic ecological modeling has matured over the last decade and leads to more predictive and flexible models, which are increasingly used for solving a wide range of applied problems (Stillman et al. 2015). We provide some guidelines on how to run bee mechanistic models to help detect critical stress combinations from large-scale monitoring program datasets. We concur with Goulson et al. (2015) that it is necessary to develop pollinator monitoring programs, but 
the field design of those programs should, whenever possible, target the capture of information on critical combinations of stressors predicted in silico.

Ecological data collected at landscape scale (i.e., hundreds of $\mathrm{km}^{2}$ ) under question-oriented monitoring surveys has propelled a reappraisal of environmental stressors in the observed decline of wild bees and honeybees. For instance, in the ECOBEE program (Odoux et al. 2014), a strong focus has been developed toward a better understanding of the relationships between honeybee colony traits and the environment. This has demonstrated that food availability appears as one of the main drivers of honeybee colony dynamics (Requier et al. 2014), and temporal limitations in food availability between mass flowering bloom events (Bretagnolle and Gaba 2015) generate strong cascading effects on colony health (Requier et al. 2016; see also Horn et al. 2016). Still, other stressors can act synergistically so that their respective effect on bee survival may be hard to discriminate in the field (Henry et al. 2014, 2015), and there is accumulating evidence that combined stresses actually exacerbate mortality in honeybees and wild bees. Excess mortality has been empirically evidenced mostly with twoway stressor interactions, including parasites $\times$ pathogens (Nazzi et al. 2012; Retschnig et al. 2014), poor nutrition $\times$ pathogens (Di Pasquale et al. 2013), pesticides $\times$ pathogens (Alaux et al. 2010; Aufauvre et al. 2012; Doublet et al. 2015; Fauser-Misslin et al. 2014), pesticides $\times$ pesticides (Vidau et al. 2011; Gill et al. 2012; Johnson et al. 2013; Henry et al. 2015), and pesticides $\times$ weather or landscape context combinations (Henry et al. 2014; Park et al. 2015). See also Collison et al. (2015) for a thorough quantitative review of pesticides $\times$ pathogens case studies.

However, the number of possible combinations increases exponentially as more candidate stressors are considered, eventually making it prohibitively expensive to cover the whole range of possibilities through traditional in vitro and in vivo approaches (Goulson et al. 2015). Alternatively, the in silico pathway provides a useful framework for combining and assessing complex stressor interactions in bees (Barron 2015). The idea of running bee models to simulate in silico any multiple-way combination of stressors has recently emerged (Becher et al. 2013), stemming from the current flourishing of literature on bee mechanistic models addressing specific issues (e.g., honeybee colonies [Schmickl and Crailsheim 2007; Becher et al. 2010, 2014; Khoury et al. 2011, 2013; Torres et al. 2015], bumblebee colonies [Bryden et al. 2013], solitary bees [Everaars and Dormann 2014]). The in silico pathway may be used to investigate tipping points under combined stressors and has provided significant improvement on the understanding of bee mortality (Henry et al. 2012; Perry et al. 2015; Horn et al. 2016; Rumkee et al. 2015). Tipping points (Figure 1) refer here to stress levels beyond which a small variation translates into a drastic constraint on the sustainability of the whole system, i.e., a steep increase in the probability to reach a given endpoint threshold (typically the bee population or colony collapse).

Some of these open source bee models are expected to be used by an increasing number of bee biologists for the study of combined stresses (Barron 2015) and recalibrated for a variety of specific bioclimatic contexts and research issues. In that respect, we believe that a coherent research agenda is urgently needed, along with a unified terminology to facilitate communication among

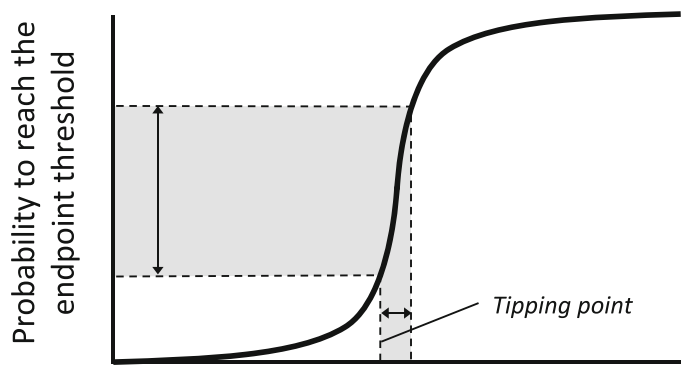

Stress level

Figure 1. Predictive systems models may be used to investigate tipping points in bee populations under environmental stresses. Tipping points denote the critical stress levels beyond which a slight increase translates into a steep change in the state of the whole system, e.g., a drastic rise of the probability to reach a given monitored endpoint threshold (double arrows). In honeybees, typical endpoints of interest are colony collapse or low population size (e.g., $<4000$ individuals at the end of the season; Becher et al. 2014; Horn et al. 2016; Rumkee et al. 2015). 
users. We do not plead however for an in silico approach as a substitute for empirical studies. Instead, it should be viewed as a tool to guide empirical studies throughout the immense scope of possibilities down to a reduced subset of plausible and testable predictions, that is to say, funneling candidate scenarios into the conception of both targeted small-scale experiments and of large field monitoring programs (e.g., the ECOBEE monitoring survey in France [Odoux et al. 2014], the German bee monitoring project [Genersch et al. 2010], the US national surveys [van Engelsdorp et al. 2008], the EPILOBEE European survey [EPILOBEE Consortium et al. 2016]).

We called this approach funnel analysis and provide here general guidelines for using it, with special emphasis on the well-documented honeybee, though the same procedure applies to models targeting other bee species. We first describe the procedure in the Material and methods section, along with an appropriate terminology. Second, by using BEEHAVE as a case study honeybee system model (Becher et al. 2014), we tested whether demographic tipping points may be identified when simulating individual stresses on important colony traits taken separately (e.g., forager mortality, larvae mortality, Varroa mite infestation, foraging activity) and whether combining those stresses would hasten the tipping point, as is expected from current hypotheses on the multifactorial nature of bee declines. We finally discuss the forthcoming opportunities for bee colony model users to coordinate their efforts for a better understanding of the environmental drivers of bee declines.

\section{MATERIAL AND METHODS}

\subsection{Prerequisite for the multistress funnel analysis}

Models, even if they are complex, are simplified representations. Therefore, to base inference about real bees on model output, all model assumptions and their justifications need to be documented so that they can be scrutinized. For example, the Overview, Design concepts, Details (ODD; Grimm et al. 2006, 2010) standard documentation of the BEEHAVE honeybee colony model (Becher et al. 2014) allowed a European expert group to evaluate all model assumptions (European Food Safety Authority 2015). Furthermore, models should be rich enough in structure and processes so that they can be empirically validated at different hierarchical levels (DeAngelis and Wolf 2003; Grimm et al. 2005). For example, Horn et al. (2016) used the BEEHAVE model to identify time periods within a foraging season where honeybee colonies are most sensitive to gaps in forage supply. They then trace these findings back to specific changes in colony structure and dynamics, which can be tested in subsequent targeted small-scale experiments. Finally, to enable testing of a model under different environmental settings, effects of the most important environmental drivers should emerge from first principles instead of imposing them via empirical rules. For honeybee models, this means making colony dynamics dependent on weather and use the availability, distribution, and foraging of nectar and pollen so that the same model can be used in different landscapes (e.g., Becher et al. 2014; Horn et al. 2016; Rumkee et al. 2015).

If a model fulfills these requirements, it can be used for what we here refer to as funnel analysis (Figure 2). For such analyses, one needs to (i) implement appropriate climate and/or landscape input model parameters for the bioclimatic context of interest and (ii) choose a relevant endpoint threshold among the bee model outputs. Simulating combined stresses on bees requires that users carefully decide what endpoint critical values are to be considered for drawing conclusions from simulations. In the case of honeybees, the typical endpoint of interest for bee biologists and beekeepers is colony survival over a season. Other endpoints may include colony size at the end of the year, peak colony size, economic value of honey stores or pollination services, or production of queens and offspring in wild bees. In general, endpoints should be used that can demonstrate thresholds and tipping points (Figure 1) as this translates the output of even complex models into a format that can directly inform management and policy development (Stillman et al. 2016).

\subsection{Essentials of the multistress funnel analysis}

The multistress funnel analysis we propose here (Figure 2) is based on a mixture of implicit and 


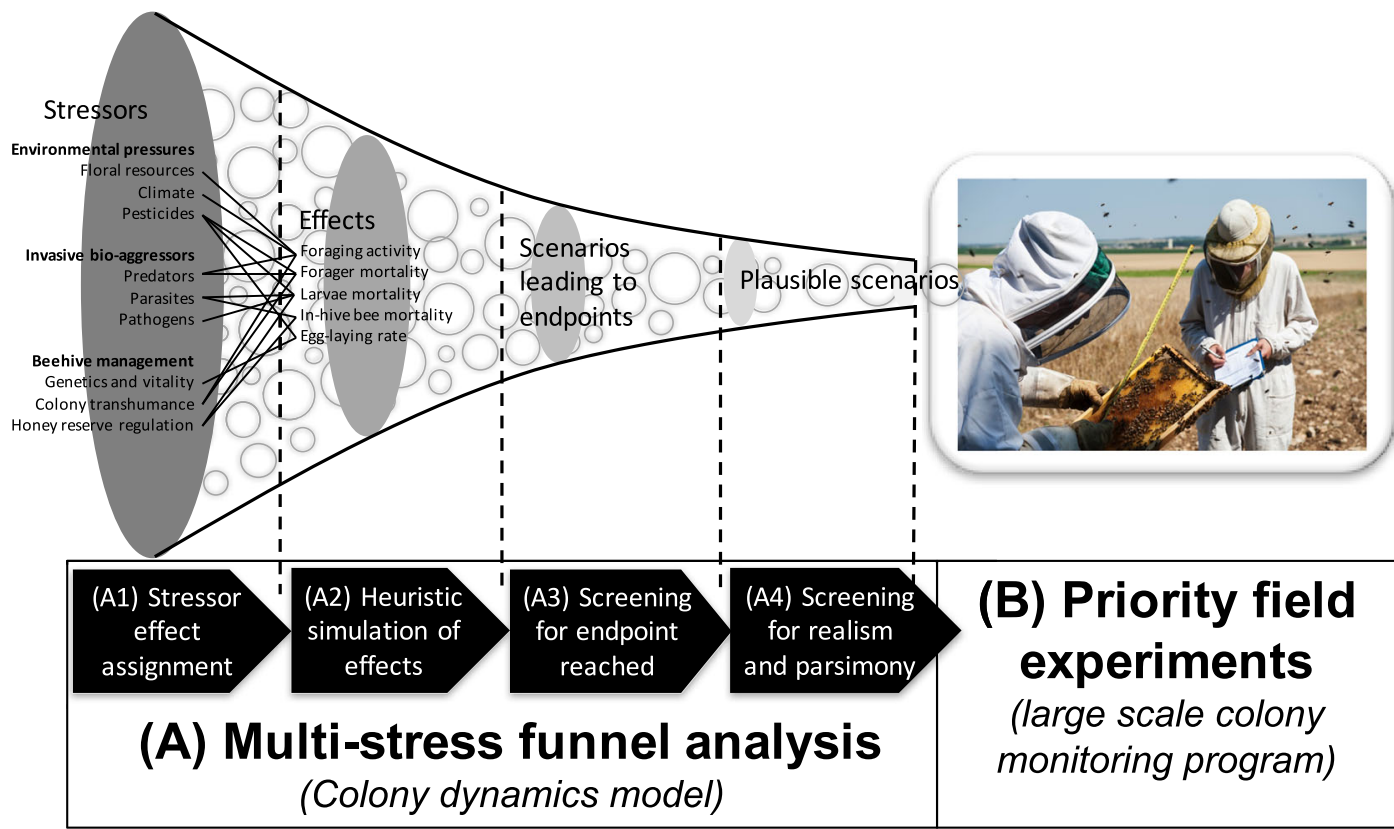

Figure 2. The multistress funnel analysis applied to honeybee colony dynamic models. In silico virtual colony (Schmickl and Crailsheim 2007; Becher et al. 2010, 2014; Khoury et al. 2011, 2013; Torres et al. 2015) simulations should sequentially drop combined stressor scenarios until delivering a limited subset of plausible scenarios testable as a part of field monitoring programs (van Engelsdorp et al. 2008; Genersch et al. 2010; Odoux et al. 2014). See Material and methods section for step-by-step details.

explicit representations of stressor effects on core honeybee colony traits. Few stressors are explicitly parameterized in current models, i.e., explicitly taken into account via specific parameters and equations. This is the case with Varroa mites and the deformed wing virus (DWV) whose infestation levels are tunable as a part of the input parameters in BEEHAVE virtual colonies (Becher et al. 2014). Distance and quantity of nectar and pollen available are also explicitly defined in BEEHAVE as well as the effects of lack of pollen and nectar on the brood (Becher et al. 2014; Horn et al. 2016). Still, many candidate honeybee stressors have to be implicitly implemented by simulating their effect on core colony life history traits, i.e., those colony traits that most influence the fate of the colony (step A1 in Figure 2). For instance, the excess mortality due to homing failure in intoxicated forager bees was implicitly implemented by inflating the foraging mortality parameter (Henry et al. 2012; Becher et al. 2014). Core colony traits may be identified by means of model sensitivity analyses (Saltelli et al. 2008; Thiele et al. 2014). Such analyses can comprise factorial designs of stressors which are then condensed into variance partitioning methods (Horn et al. 2016) or via screening for the most sensitive parameters and then exploring, via global analysis, the relative importance of these processes and their interactions. Typical core colony traits concern the baseline demographic functioning of the colony (e.g., brood mortality, in-hive workers, and foragers mortality; Schmickl and Crailsheim 2007; Becher et al. 2014; Rumkee et al. 2015).

Therefore, the funnel analysis approach we recommend here actually consists of simulating variations of one or multiple core colony traits and identifying which scenarios may lead to the endpoint thresholds of interest (steps $\mathrm{A} 2$ and $\mathrm{A} 3$ in Figure 2) and then which ones are realistic enough, in terms of stress realism and parsimony, to be tested in the field (steps A4 and B in Figure 2). Those steps are further detailed below and illustrated in Figure 2: 
(A) From the full scope of possibilities down to the most plausible subset of critical stress combinations.

(A1) Stressor effect assignment: identifying from literature and expert knowledge a qualitative network of effects linking the candidate stressors (e.g., nosema spores, neonicotinoid pesticides, floral resource scarcity) with the affected core colony traits (e.g., foraging intensity, brood mortality, in-hive workers, and foragers mortality).

(A2) Heuristic simulation of effects on core colony traits: simulating all possible combinations of core colony trait variations. Trait variations may be either persistent throughout the season or restricted to a particular period. Likewise, the magnitude of trait variations may be allowed to vary within a realistic range. One may compute broad variation scenarios (e.g., low, medium, or large effect size) or series of scenarios with finer resolution depending on computational performance.

(A3) Screening candidate scenarios for specific goals and endpoint criteria: The candidate scenarios that result in a high probability of colonies reaching the predetermined endpoint threshold of interest are singled out. The threshold probability for retaining and rejecting scenarios is decided before the analyses, based on goals for protection of colonies or risk deemed acceptable. One example of specific goals being applied is in the pesticide regulatory arena. In line with the European Food Safety Authority guidance for acceptable honeybee protection goals, Rumkee et al. (2015) proposed the lethal imposed stress $\left(\mathrm{LIS}_{\mathrm{x}}\right)$ framework for colonies, analogous to the standard lethal dose $\left(\mathrm{LD}_{50}\right)$ currently used for pesticide risk assessment on an individual basis. The $\mathrm{LIS}_{50}$ (or LIS 10 ) indicates the level of imposed stress resulting in $50 \%$ (or $10 \%$ ) probability of colonies reaching a chosen endpoint threshold. Using such a framework would screen out the scenarios that predict less than a $50 \%$ (or $10 \%$ ) chance of reaching the chosen threshold. Alternatively, a cutoff rule may be based on the statistical power of the envisioned field monitoring, i.e., the finest detectable change of endpoint threshold occurrence frequency given actual sample size.

(A4) Screening candidate scenarios for realism and parsimony criteria: Within the subset of candidate scenarios meeting the endpoint criteria, some are likely to be more realistic than others considering stressor temporal or spatial co-occurrence. Expert knowledge is required at this step to decide which scenario should receive priority given field realism and parsimony of stressor combinations. Experts may set aside candidate scenarios with unrealistically high stress levels or with stressors occurring at inappropriate periods of the season. For instance, some experts in a given eco-region may be primarily concerned by the effects of increased forager mortality due to neonicotinoid residuals during oilseed rape blooms and of inhibited pollen foraging activity due to the presence of attacking yellow-legged hornets at hive entrance. Those stresses occur, respectively, in spring (Henry et al. 2015) and in early fall (Rome et al. 2015). Experts may therefore recommend focusing on the corresponding simulations with acute forager mortality in spring and reduced foraging activity in fall in order to further assess potential associations with other stressors throughout the season, such as pathogens or climate hazards. Conversely, experts may judge it unlikely that all life stages (eggs, larvae, pupae, in-hive workers, established foragers) be simultaneously subject to acute mortality. For the sake of parsimony, they may recommend selecting simulations with only one or two life stages being simultaneously affected by acute mortality levels.

\section{(B) Priority field experiments.}

The most plausible and parsimonious scenarios may finally be addressed in the field, either by suggesting new hypotheses or novel dataset crosscomparisons to be investigated in already existing large-scale monitoring programs. Field (in vivo) monitoring surveys should be aimed at confirming the links between endpoint threshold occurrence and core colony trait alterations. Laboratory (in vitro) experiments may further help untangle the explicit links with specific stressors. Regardless 
of the chosen monitoring design, time series analytical approaches would be requested, whereby correlative links would be tested between the presence or magnitude of candidate stressors, the core colony traits at a given time of the season, and the subsequent state of colonies later on (endpoint threshold reached or not).

\subsection{Assessing combined effects of multiple stressors}

To reveal critical stressor combinations, predictive systems models should be able to reveal combined effects, i.e., they should predict that multiple stressors produce a greater effect than individual stressors taken separately. To test this assertion, we compared in silico the fate of honeybee colonies with a series of stresses set at the tipping point and simulated either separately or in combinations. Although purely illustrative, this simple example shows how independent stressors can combine their respective effect to promote colony failure.

Initial settings We used the BEEHAVE model (Becher et al. 2014) with Horn's et al. (2016) initial colony settings, and we implemented a typical weather and landscape dataset from the ECOBEE colony monitoring area (Odoux et al. 2014). We allowed a baseline infestation with 100 virus-infected Varroa mites on the first day of simulation as default value. Current beekeeping practices were also simulated with the ad hoc options (default settings for Varroa treatment and honey harvests, see Becher et al. 2014).

Endpoint Colony sizes smaller than 4000 adult bees at the end of the year (Becher et al. 2014; Horn et al. 2016; Rumkee et al. 2015) were deemed insufficient for sustainable honey production and survival throughout the beekeeping season without human intervention. We therefore used this demographic threshold as an endpoint value to illustrate the combined stress analysis. Colony simulations were run for 3 years or until the endpoint threshold was reached.

Core colony traits and tipping points We focused on four core colony traits that are thought to have profound impact on demographic trajectories: forager mortality, larvae mortality, virus-infected Varroa mites, and reduction of the foraging activity. We performed a sensitivity analysis to identify critical tipping points associated with those core colony traits taken individually. Default model parameters were gradually increased (larvae and forager mortality rate, Varroa infestation) or decreased (maximal foraging distance allowed) to delineate the endpoint threshold occurrence probability with increasing stress values. First, exploratory simulations were run by series of 10 repetitions, until detecting the range of stress values leading to low $(n=1)$ and high $(n=10)$ endpoint threshold occurrence. The range was then systematically covered by 20 series of 10 simulation repetitions for a better resolution. Simulation outputs were finally fitted with a standard logistic function by using a generalized linear model (GLM) for binomial data ( $\mathrm{glm}$ function in $\mathrm{R}$ software for statistical computing, R Core Team 2014). The tipping point level was taken to be the highest stress value the simulated colonies could afford without reaching the endpoint threshold.

Stress implementation We tested the hypothesis that combining two or more stresses at their tipping point (the others being set to the default value) would precipitate colonies to endpoint, which would normally not occur with a single stressor. All multiple-way tipping point combinations $(n=16)$ were simulated 20 times each, totaling 320 simulated colonies. The probability to reach the endpoint threshold out of the 20 simulations was computed and compared by using simple a posteriori pairwise exact binomial tests among stress combinations (binom.test function, R Core Team 2014).

\section{RESULTS}

Core colony traits and tipping points A tipping point pattern was successfully established when tuning each of the four studied core colony traits (Figure 3) and satisfactorily modeled by using logistic functions (Table I). Estimated tipping points were 1.42 for forager mortality rate per $10^{5} \mathrm{~s}$ foraging (default $\left.=1\right), 0.06$ for daily larval mortality rate (default $=0.01$ ), 1142 for initial number of infected mites (default $=100$ ), and $3 \mathrm{~km}$ for maximal distance flown by foragers on a single day (default limited by duration of clement weather for foraging).

Stress implementation Of the 320 simulated colonies covering the 16 possible tipping point 


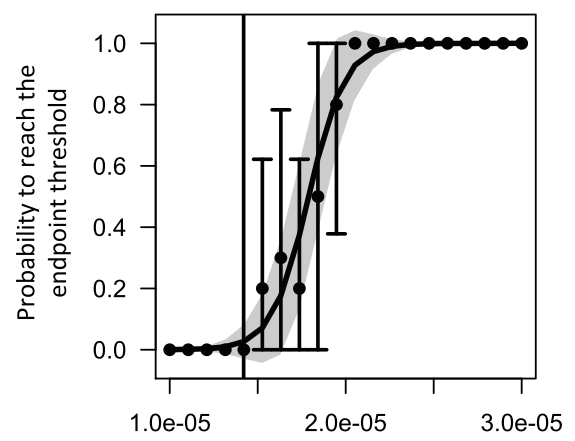

Forager mortality rate (per s)

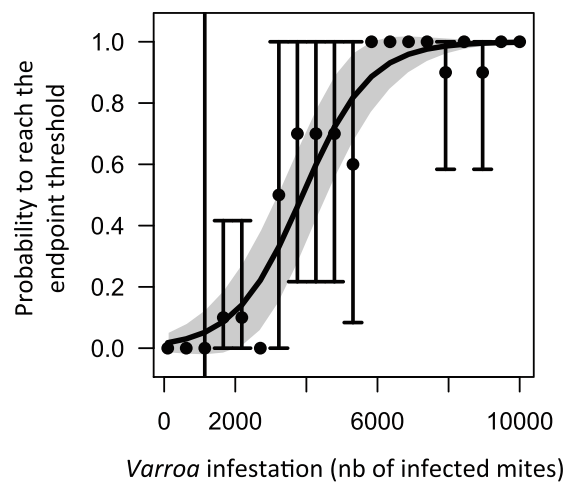

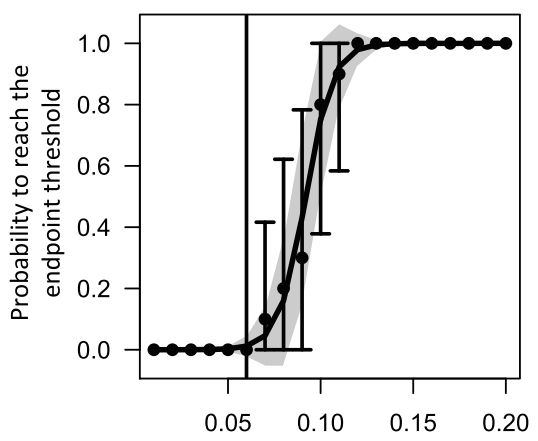

Larval mortality rate (per day)

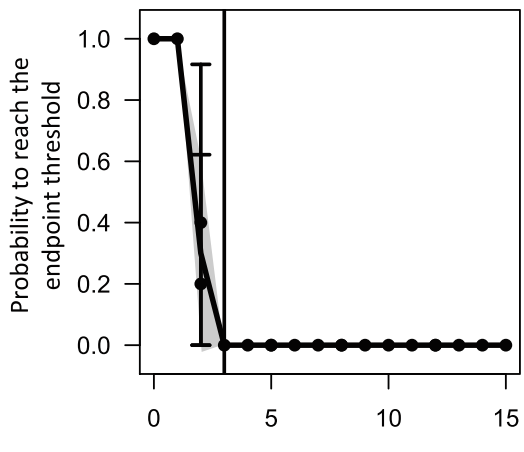

Maximal foraging distance $(\mathrm{km})$

Figure 3. Assessment of tipping points associated with four core colony traits. The probability to reach the endpoint threshold ( $<4000$ adult honeybees) dramatically increases beyond a critical stress value referred to as tipping points (vertical line). Dots and bars stand for the mean \pm SD endpoint threshold occurrence per series of 10 replicated colony simulations. Trends are depicted by a logistic fit to simulation data (thick lines and $95 \%$ confidence limit as shaded areas, see Table I for details).

combinations, 14 collapsed or fell below the endpoint threshold in the course of the first year, 120 in the second year, and 15 in the third year. Simulation outputs merely illustrate the combined stressor hypothesis developed in current scientific literature (Figure 4). Taken alone, stressors at tipping point levels did not precipitate simulated colonies to reach the endpoint threshold. For instance, colonies with a $42 \%$ increase in forager mortality were already on the brink of reaching

Table I. Logistic model statistics for predicting of the probability to reach the endpoint threshold as a function of stress level imposed on four core colony traits.

Core colony trait value $x$

Forager mortality rate (per $10^{5} \mathrm{~s}$ foraging)

Larval mortality rate (per day)

Varroa infestation (nb. of infected mites)

Foraging activity (maximal daily foraging distance, $\mathrm{km}$ ) $\alpha$

$\beta$

Chi-squared statistics for deviance

The predicted endpoint curves (Figure 3) are of the form $p_{\text {Endpoint }}=1 /\left(1+e^{-(\alpha x+\beta)}\right)$, with $x$ the focus colony trait value and $\alpha$ and $\beta$ the logistic model estimates returned by GLMs 


\begin{tabular}{|c|c|c|c|}
\hline \multicolumn{4}{|l|}{$x$} \\
\hline & $x$ & & \\
\hline & & $x$ & \\
\hline & & & $x$ \\
\hline & & $x$ & $x$ \\
\hline$x$ & & & $x$ \\
\hline$x$ & & $x$ & $x$ \\
\hline \multirow[t]{2}{*}{$x$} & $x$ & & \\
\hline & $x$ & & $x$ \\
\hline \multirow[t]{2}{*}{$x$} & & $\times$ & \\
\hline & $x$ & $x$ & \\
\hline \multirow[t]{2}{*}{$x$} & $x$ & & $x$ \\
\hline & $x$ & $x$ & $x$ \\
\hline$\times$ & $\times$ & $\times$ & \\
\hline$x$ & $x$ & $x$ & $x$ \\
\hline $\begin{array}{c}\text { Forager } \\
\text { mortality }\end{array}$ & $\begin{array}{l}\text { Larvae } \\
\text { mortality }\end{array}$ & $\begin{array}{c}\text { Varroa } \\
\text { pressure }\end{array}$ & $\begin{array}{c}\text { Foraging } \\
\text { activity }\end{array}$ \\
\hline
\end{tabular}

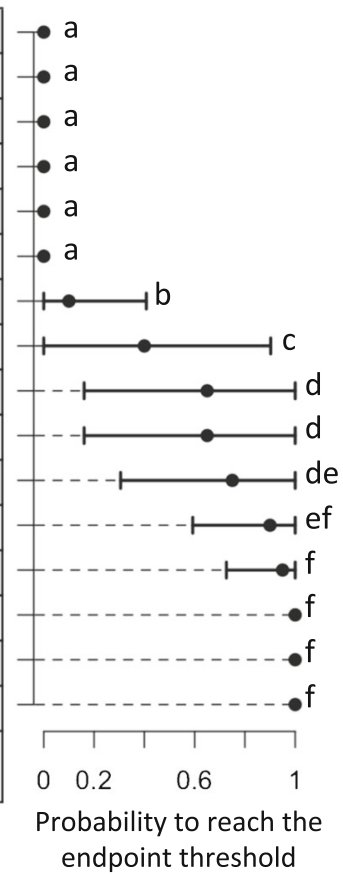

Figure 4. Comparison of combined stress scenarios for endpoint criteria. For each of the 16 simulated combined stress scenarios (left panel), the risk to reach the critical endpoint threshold (right panel) is given as a probability value $( \pm \mathrm{SD})$. Probability values that are identified by distinct letters in the right panel are significantly different from each other (a posteriori exact binomial tests among scenarios of combined stresses, see text).

the endpoint threshold, but regulation mechanisms in the colony compensated these forager losses. However, the two-way combinations were associated with a range of significantly higher endpoint threshold occurrences (10 to $90 \%$, Figure 4), confirming the model ability to identify sharp combined effects contrasting with separate effects of stresses taken alone. With three or more combined stresses, the endpoint is almost inevitably reached (>95\%, Figure 4).

Toward funnel analysis This demonstrates that tuning only a couple of core honeybee colony parameters can result in a broad range of collapse scenarios. In practice, the funnel analysis is indeed intended to single out which of those scenarios should receive priority in question-oriented field monitoring programs. From this simplistic hypothetical example (Figure 4), the funnel process would first screen out the scenarios that had no chance to lead to the endpoint of interest $(n=6$ scenarios). Then, experts may decide to screen out scenarios with a $10 \%$ endpoint probability cutoff rule ( $n=1$ scenario), given that this approximates their maximal field monitoring statistical resolution (see also the $\mathrm{LIS}_{50}$ framework in Rumkee et al. 2015). They may further decide to screen out scenarios with altered foraging activity ( $n=5$ scenario) because they may consider that this level of stress - maximal daily foraging distance limited down to $3 \mathrm{~km}$ per forager only-is unrealistically restrictive. Having reduced candidate scenarios from 16 down to 4 , experts may finally adjust their field study so as to target the possible environmental drivers of larval mortality, which delivers the highest endpoint threshold probabilities when combined with Varroa mites. 


\section{DISCUSSION}

The idea underlying the suggested multistress funnel analysis is to funnel candidate scenarios into explicit field monitoring programs while screening for endpoint criteria and for realism and parsimony of candidate stressor combinations. Having confirmed that bee systems models can reveal critical multistress combinations, we recommend that future research investigates the ability of funnel analyses to effectively end up in a small subset of candidate scenarios. There is no guarantee that it will invariably succeed, but it will at least help identify the most influential factors and discover unexpected ones in the studied systems.

Sensitivity to certain stressors is likely to depend on eco-region, weather, landscape structure, and management, so that focusing monitoring and experiments on the wrong factors in a certain eco-region might lead to enormous waste of resources and time. The ultimate aim of model-based funnel analysis is to screen the thousands of conceivable scenarios of combined stressors down to a short list of plausible scenarios humanly manageable in small-scale experiments and field monitoring programs. In that respect, we fully support Goulson et al.'s (2015) call for developing pollinator monitoring programs, but we further encourage bee biologists to conceive or adjust those programs considering mechanistic model predictions of what might be the most critical stress combinations for the considered endpoint. With this in mind, we expect higher colony resilience in social bees owing to buffering mechanisms as a consequence of colony living and division of labor (Henry et al. 2015; Rundlöf et al. 2015). This makes social bees also a testbed for testing theories and approaches regarding resilience and tipping points. With managed honeybee colonies, for example, we can, in contrast to full ecosystems, manipulate buffer mechanisms and study recovery rates and the ability of colonies to absorb disturbances and maintain functioning. This is also in line with the plea for a more mechanistic and predictive "systems ecology" (Evans et al. 2013). Solitary wild bees are likely to be more susceptible and hence might provide even more sensitive model organisms to test combined stresses (European Academies Science Advisory Council 2015; Rundlöf et al. 2015), but with different resilience mechanisms.

Bee biologists are now at a crossroads of action and reflection. Although efforts should be spent in the development of operative solutions for sustainable beekeeping and maintaining viable wild bee populations (Goulson et al. 2015), disentangling the respective effects of the many candidate stressors at the roots of bee decline is still required. Simulating virtual bee systems is one promising tool in that respect. Mechanistic models of complex biological systems cannot be viewed as perfectly reflecting reality. However, even though a given model may be lacking empirical support for a specific context of interest, we would not discourage bee biologists from investigating the model simulation outputs as a part of their research, providing they keep in mind that the outputs are conditional predictions, i.e., predictions that are dependent on the assumptions the model is based on. Model assumptions, formalized by a series of parameters and equations, will gain accuracy as developers and users gain empirical knowledge. At the same time, the next steps in model development will be (i) to develop specific modules for interaction effects among stressors, as is currently evidenced in laboratory but still poorly considered in predictive systems models and (ii) to move beyond the dynamics of the colony to the dynamics of the actual populations, so that the densities of managed colonies or native species can be predicted over time and space. Using funnel analysis to match model simulations with empirical work will lead to deeper insight into the mechanisms behind broader scale patterns of colony and even species loss. 


\section{ACKNOWLEDGMENTS}

This work was supported by the European Community program (797/2004) for French beekeeping coordinated by the French Ministry of Agriculture (RISQAPI project). JLO, MAB, and PK were funded by grants from the Biotechnology and Biological Sciences Research Council, UK [BB/J014915/1; BB/ K014463/1]. We thank Axel Decourtye and JeanFrançois Odoux for useful discussions on honeybee colony modeling and field monitoring design, as well as two anonymous reviewers for constructive comments on the manuscript.

Author contributions Mickaël Henry, Matthias A. Becher, Juliet L. Osborne, Peter J. Kennedy, Pierrick Aupinel, Vincent Bretagnolle, François Brun, Volker Grimm, Juliane Horn, and Fabrice Requier contributed to the design and writing of the study. FR and JH performed the simulations.

Les modèles prédictifs de systèmes peuvent contribuer à élucider les phénomènes de déclin des abeilles soumises à des stress multiples

Apis mellifera / programme de surveillance / abeilles mellifères / modélisation mécaniste / modèles agentbasés

Vorhersagemodelle können helfen, den Rückgang der Bienen aufgrund multipler Stressoren aufzuklären

apis mellifera / feldmonitoring / honigbienen / mechanistische modellierung / agentenbasierte modelle

\section{REFERENCES}

Alaux, C., Brunet, J.-L., Dussaubat, C., Mondet, F., Tchamitchan, S., Cousin, M., Brillard, J., Baldy, A., Belzunces, L.P., Conte, Y.L. (2010) Interactions between Nosema microspores and a neonicotinoid weaken honeybees (Apis mellifera). Environ. Microbiol. 12, 774-782

Aufauvre, J., Biron, D.G., Vidau, C., Fontbonne, R., Roudel, M., Diogon, M., Viguès, B., Belzunces, L.P., Delbac, F., Blot, N. (2012) Parasite-insecticide interactions: a case study of Nosema ceranae and fipronil synergy on honeybee. Sci. Rep. 2, 326

Barron, A.B. (2015) Death of the bee hive: understanding the failure of an insect society. Curr. Opin. Insect Sc. 10, 45-50

Becher, M.A., Hildenbrandt, H., Hemelrijk, C.K., Moritz, R.F.A. (2010) Brood temperature, task division and colony survival in honeybees: a model. Ecol. Model. 221, 769-776

Becher, M.A., Osborne, J.L., Thorbek, P., Kennedy, P.J., Grimm, V. (2013) Towards a systems approach for understanding honeybee decline: a stocktaking and synthesis of existing models. J. Appl. Ecol. 50, $868-880$

Becher, M.A., Grimm, V., Thorbek, P., Horn, J., Kennedy, P.J., Osborne, J.L. (2014) BEEHAVE: a systems model of honeybee colony dynamics and foraging to explore multifactorial causes of colony failure. J. Appl. Ecol. $\mathbf{5 1}, 470-482$

Bretagnolle, V., Gaba, S. (2015) Weeds for bees? A review. Agron. Sustain. Dev. 35, 891-909

Bryden, J., Gill, R.J., Mitton, R.A.A., Raine, N.E., Jansen, V.A.A. (2013) Chronic sublethal stress causes bee colony failure. Ecol. Lett. 16, 1463-1469

Collison, E., Hird, H., Cresswell, J., Tyler, C. (2015) Interactive effects of pesticide exposure and pathogen infection on bee health - a critical analysis. Biol. Rev. in press, doi: 10.1111/brv.12206

R Core Team (2014) R: a language and environment for statistical computing. R Foundation for Statistical Computing, Vienna

DeAngelis, D.L., Wolf, M. (2003) In praise of mechanistically rich models, in : Canham, C.D., Cole, J.J., Lauenroth, W.K. (Eds.), Models in ecosystem science, Princeton University Press, Princeton, pp. 63-82

Di Pasquale, G., Salignon, M., Le Conte, Y., Belzunces, L.P., Decourtye, A., Kretzschmar, A., Suchail, S., Brunet, J.-L., Alaux, C. (2013) Influence of pollen nutrition on honey bee health: do pollen quality and diversity matter? PLoS One 8, e72016

Doublet, V., Labarussias, M., de Miranda, J.R., Moritz, R.F.A., Paxton, R.J. (2015) Bees under stress: sublethal doses of a neonicotinoid pesticide and pathogens interact to elevate honey bee mortality across the life cycle. Environ. Microbiol. 17, 969-983

EPILOBEE Consortium, Chauzat, M.-P., Jacques, A., Laurent, M., Bougeard, S., Hendrikx, P., RibièreChabert, M. (2016) Risk indicators affecting honeybee colony survival in Europe: one year of surveillance. Apidologie, 47, 348-378.

European Academies Science Advisory Council. (2015) Ecosystem Services, Agriculture and Neonicotinoids, EASAC policy report 26, Halle, Germany

European Food Safety Authority. (2015) Statement on the suitability of the BEEHAVE model for its potential use in a regulatory context and for the risk assessment of multiple stressors in honeybees at the landscape level. EFSA Journal 13, 4125:4216

Evans, M.R., Bithell, M., Cornell, S.J., Dall, S.R.X., Díaz, S., et al. (2013) Predictive systems ecology. Proc. R. Soc. B 280, 20131452

Everaars, J., Dormann, C.F. (2014) Simulation of olitary (non-Apis ) bees competing for pollen, in Devillers, J. (Ed.), in Silico Bees, CRC Press, Taylor, Francis Group, Boca Raton, pp. 209-268 
Fauser-Misslin, A., Sadd, B.M., Neumann, P., Sandrock, C. (2014) Influence of combined pesticide and parasite exposure on bumblebee colony traits in the laboratory. J. Appl. Ecol. 51 , 450-459

Genersch, E., von der Ohe, W., Kaatz, H., Schroeder, A., Otten, C., et al. (2010) The German bee monitoring project: a long term study to understand periodically high winter losses of honey bee colonies. Apidologie 41, 332-352

Gill, R.J., Ramos-Rodriguez, O., Raine, N.E. (2012) Combined pesticide exposure severely affects individual- and colony-level traits in bees. Nature 491, 105-108

Goulson, D., Nicholls, E., Botías, C., Rotheray, E.L. (2015) Bee declines driven by combined stress from parasites, pesticides, and lack of flowers. Science 347, 1255957

Grimm, V., Revilla, E., Berger, U., Jeltsch, F., Mooij, W.M., Railsback, S.F., Thulke, H.-H., Weiner, J., Wiegand, T., DeAngelis, D.L. (2005) Pattern-oriented modeling of agent-based complex systems: lessons from ecology. Science 310, 987-991

Grimm, V., Berger, U., Bastiansen, F., Eliassen, S., Ginot, V., et al. (2006) A standard protocol for describing individual-based and agent-based models. Ecol. Model. 198, 115-126

Grimm, V., Berger, U., DeAngelis, D.L., Polhill, J.G., Giske, J., Railsback, S.F. (2010) The ODD protocol: a review and first update. Ecol. Model. 221 , 2760-2768

Henry, M., Béguin, M., Requier, F., Rollin, O., Odoux, J.F., Aupinel, P., Aptel, J., Tchamitchian, S., Decourtye, A. (2012) A common pesticide decreases foraging success and survival in honey bees. Science $\mathbf{3 3 6}$, 348-350

Henry, M., Bertrand, C., Le Féon, V., Requier, F., Odoux, J.F., Aupinel, P., Bretagnolle, V., Decourtye, A. (2014) Pesticide risk assessment in free-ranging bees is weather and landscape dependent. Nat. Commun. 5, 4359

Henry, M., Cerrutti, N., Aupinel, P., Decourtye, A., Gayrard, M., Odoux, J.-F., Pissard, A., Rüger, C., Bretagnolle, V. (2015) Reconciling laboratory and field assessments of neonicotinoid toxicity to honeybees. Proc. R. Soc. B 282, 20152110

Horn, J., Becher, M.A., Kennedy, P.J., Osborne, J.L., Grimm, V. (2016) Multiple stressors: using the honeybee model BEEHAVE to explore how spatial and temporal forage stress affects colony resilience. Oikos 125, 1001-1016

Johnson, R.M., Dahlgren, L., Siegfried, B.D., Ellis, M.D. (2013) Acaricide, fungicide and drug interactions in honey bees (Apis mellifera). PLoS One 8, e54092

Khoury, D.S., Myerscough, M.R., Barron, A.B. (2011) A quantitative model of honey bee colony population dynamics. PLoS One 6 e18491

Khoury, D.S., Barron, A.B., Myerscough, M.R. (2013) Modelling food and population dynamics in honey bee colonies. PLoS One 8, e59084

Nazzi, F., Brown, S.P., Annoscia, D., Del Piccolo, F., Di Prisco, G., Varricchio, P., Della Vedova, G., Cattonaro, F., Caprio, E., Pennacchio, F. (2012) Synergistic parasite-pathogen interactions mediated by host immunity can drive the collapse of honeybee colonies. PLoS Pathog. 8, e1002735

Odoux, J.-F., Aupinel, P., Gateff, S., Requier, F., Henry, M., Bretagnolle, V. (2014) ECOBEE: a tool for long-term bee colony monitoring at landscape scale in west European intensive agrosystems. J. Apic. Res. 53 , 57-66

Park, M.G., Blitzer, E.J., Gibbs, J., Losey, J.E., Danforth, B.N. (2015) Negative effects of pesticides on wild bee communities can be buffered by landscape context. Proc. R. Soc. B 282, 20150299

Perry, C.J., Søvik, E., Myerscough, M.R., Barron, A.B. (2015) Rapid behavioral maturation accelerates failure of stressed honey bee colonies. Proc. Natl. Acad. Sc. U.S.A. doi: 10.1073/pnas.1422089112

Potts, S.G., Biesmeijer, J.C., Kremen, C., Neumann, P., Schweiger, O., Kunin, W.E. (2010) Global pollinator declines: trends, impacts and drivers. Trends Ecol. Evol. 25, 345-353

Requier, F., Odoux, J.-F., Tamic, T., Moreau, N., Henry, M., Decourtye, A., Bretagnolle, V. (2014) Honey bee diet in intensive farmland habitats reveals an unexpectedly high flower richness and a major role of weeds. Ecol. Appl. 25, 881-890

Requier, F., Odoux, J.F., Henry, M., Bretagnolle, V. (2016) The carry-over effects of spring pollen shortage negatively impact the colony dynamics and survival of managed honeybees. J. Appl. Ecol, in press

Retschnig, G., Williams, G.R., Mehmann, M.M., Yañez, O., de Miranda, J.R., Neumann, P. (2014) Sex-specific differences in pathogen susceptibility in honey bees (Apis mellifera). PLoS One 9, e85261

Rome, Q., Muller, F.J., Touret-Alby, A., Darrouzet, E., Perrard, A., Villemant, C. (2015) Caste differentiation and seasonal changes in Vespa velutina (Hym.: Vespidae) colonies in its introduced range. J. Appl. Entomol. 139, 771-782

Rumkee, J.C.O., Becher, M.A., Thorbek, P., Kennedy, P.J., Osborne, J.L. (2015) Predicting honeybee colony failure: using the BEEHAVE model to simulate colony responses to pesticides. Environ Sci. Technol. 49, 12879-12887

Rundlöf, M., Andersson, G.K.S., Bommarco, R., Fries, I., Hederström, V., et al. (2015) Seed coating with a neonicotinoid insecticide negatively affects wild bees. Nature 521, 77-80

Saltelli, A., Ratto, M., Andres, T., Campolongo, F., Cariboni, J., Gatelli, D., Saisana, M., Tarantola, S. (2008) Global sensitivity analysis: the primer, John Wiley Sons, Chichester.

Schmickl, T., Crailsheim, K. (2007) HoPoMo: a model of honeybee intracolonial population dynamics and resource management. Ecol. Model. 204, 219-245

Stillman, R.A., Railsback, S.F., Giske, J., Berger, U., Grimm, V. (2015) Making predictions in a changing world: the benefits of individual-based ecology. BioScience, 65, 140-150

Stillman, R.A., Wood, K.A., Goss-Custard, J.D. (2016) Deriving simple predictions from complex models to 
support environmental decision-making. Ecol. Model., 10.1016/j.ecolmodel.2015.04.014. in press

Thiele, J.C., Kurth, W., Grimm, V. (2014) Facilitating parameter estimation and sensitivity analysis of agentbased models: a cookbook using NetLogo and "R". JASSS 17, 11. doi:10.18564/jasss.2503

Torres, D.J., Ricoy, U.M., Roybal, S. (2015) Modeling honey bee populations. PLoS One 10, e0130966

van Engelsdorp, D., Hayes, J., Jr., Underwood, R.M., Pettis, J. (2008) A survey of honey bee colony losses in the U.S., fall 2007 to spring 2008. PLoS One 3, e4071

Vanbergen, A.J., the Insect Pollinators Initiative. (2013) Threats to an ecosystem service: pressures on pollinators. Front. Ecol. Environ. 11 , 251-259

Vidau, C., Diogon, M., Aufauvre, J., Fontbonne, R., Viguès, B., et al. (2011) Exposure to sublethal doses of fipronil and thiacloprid highly increases mortality of honeybees previously infected by Nosema ceranae. PLoS One 6, e21550 